\title{
Dimensional crossover in magnetic warwickites
}

\author{
R. B. Guimarães, J. C. Fernandes, and M. A. Continentino \\ Instituto de Física, Universidade Federal Fluminense Campus da Praia Vermelha, \\ Niterói, 24.210-340, RJ, Brazil
}

H. A. Borges

Departamento de Física, Pontifícia Universidade Católica RJ, Rio de Janeiro, 22452-970, RJ, Brazil

C. S. Moura, J. B. M. da Cunha, and C. A. dos Santos

Instituto de Física, Universidade Federal do Rio Grande do Sul, Av. Bento Gonçalves, 9500, Porto Alegre, 91501-970, RS, Brazil

(Received 27 January 1997)

\begin{abstract}
We present an investigation of the magnetic properties of a series of warwickites with different transition metals. These materials are characterized by one-dimensional structures called ribbons where the transition metals are randomly located. The present results allow for a systematic investigation of the hierarchy of magnetic interactions in these systems. While the intraribbon interactions are mainly direct $d$ - $d$ interactions between the transition-metal ions, the interribbon interaction is due to superexchange between metals in different octahedra mediated by the oxygen in the common corner. [S0163-1829(97)00625-5]
\end{abstract}

\section{INTRODUCTION}

The warwickites have been the subject of several investigations in the last years. ${ }^{1-5}$ These systems are mixed borates with general formula $M^{2+} M^{3+} \mathrm{OBO}_{3}$ which have linear structures in the form of ribbons extending along the $c$ axis (see Fig. 1). The ribbons are formed by four columns of edge-sharing oxygen octahedra in which centers are located divalent and trivalent metallic ions $\left(M^{2+}, M^{3+}\right.$, respectively). These ribbons act from the magnetic, ${ }^{1,2}$ as well as electronic point of view, ${ }^{4,5}$ as independent entities and this is responsible for the low-dimensional properties of the warwickites. Another feature of these structures is that the cation sites are randomly occupied by the divalent and trivalent metals. ${ }^{1}$ In $\mathrm{MgScOBO}_{3}$ the trivalent ions $\mathrm{Sc}^{3+}$ preferentially occupy (76\%) the inner columns 1 and $1^{\prime}$ (Ref. 6) (see Fig. 1). Consequently, besides its one-dimensional character, the warwickites are naturally disordered systems and this should be taken into account in understanding both their transport and magnetic properties. Furthermore the warwickites can be formed with most of the transition metals ${ }^{7}$ allowing for systematic investigations of their physical properties.

One-dimensional magnetic materials are as a rule idealizations in nature. Eventually at sufficiently low temperatures weak interactions between the low-dimensional structures will set in producing a crossover to higher dimensions in general accompanied by the appearance of long-range magnetic order. A previous study of the magnetic properties of $\mathrm{MgTiOBO}_{3}$ has shown that this warwickite represents a physical realization of the random exchange Heisenberg antiferromagnetic chain (REHAC), the Ti ions in the $3 d^{1}$ configuration providing the spins $S=1 / 2$. $^{1}$ This was further supported by specific-heat measurements which have shown that down to the lowest temperature of the experiments, $T=0.2$ $\mathrm{K}$, this material behaves as a REHAC with no phase transition. ${ }^{3}$ In this case the contribution for the specific heat from the low-energy magnetic excitations associated with the random singlet phase of REHAC's could be directly observed. These excitations give rise to a characteristic temperature dependence of the specific heat $C_{p} \propto T^{1-\alpha}$ where $\alpha$ is the same exponent describing the divergence of the low-temperature susceptibility, $\chi \propto T^{-\alpha}{ }^{1-3}$

Later studies on another warwickite $\mathrm{MgVOBO}_{3}\left(\mathrm{~V}^{3+}\right.$, $\left.3 d^{2}, S=1\right)$ revealed the existence of a spin-glass transition at approximately $T_{\mathrm{sg}}=6 \mathrm{~K} .^{2}$ The behavior of the susceptibility for $T>T_{\text {sg }}$ is that expected for a spin-1 REHAC, i.e., $\chi \propto T^{-\alpha}$. The transition is interpreted as caused by interribbon interactions leading to three-dimensional spin-glass ordering. These interribbon interactions are due to the superexchange between cations inside oxygen octahedra sharing a corner. A typical distance between cations in this case is $3.36 \AA$ (see Fig. 1 and Table I) so that the metals in different ribbons are too far apart for direct $d$ - $d$ overlap to be significant. This is not the case however inside the ribbons. Although the oxygens in the shared edge of the octahedra mediate superexchange interactions between metals in the same ribbon, the short cation-cation distances $\left(d_{2}=2.9 \AA\right)$ makes the direct $d$ - $d$ interaction the dominant intraribbon interaction (Fig. 2).

In this paper we present results on the magnetic properties of four warwickites namely, $\mathrm{MgCrOBO}_{3}, \mathrm{MgFeOBO}_{3}$, $\mathrm{ScNiOBO}_{3}$, and $\mathrm{ScMnOBO}_{3}$. In the first two $\mathrm{Mg}$ is divalent and $\mathrm{Cr}$ and $\mathrm{Fe}$ are trivalents with $S=3 / 2$ and $S=5 / 2$, respectively. In the next two Sc is trivalent and consequently we have $\mathrm{Ni}^{2+}$ with $S=1$ and $\mathrm{Mn}^{2+}$ with $S=5 / 2$.

\section{EXPERIMENTAL RESULTS}

The samples were powdered prepared using the same procedure of our previous studies ${ }^{1,2}$ except for $\mathrm{MgFeOBO}_{3}$ which was prepared using borax as flux. They were all characterized by $\mathrm{x}$-ray powder diffraction (XRPD) by using a 


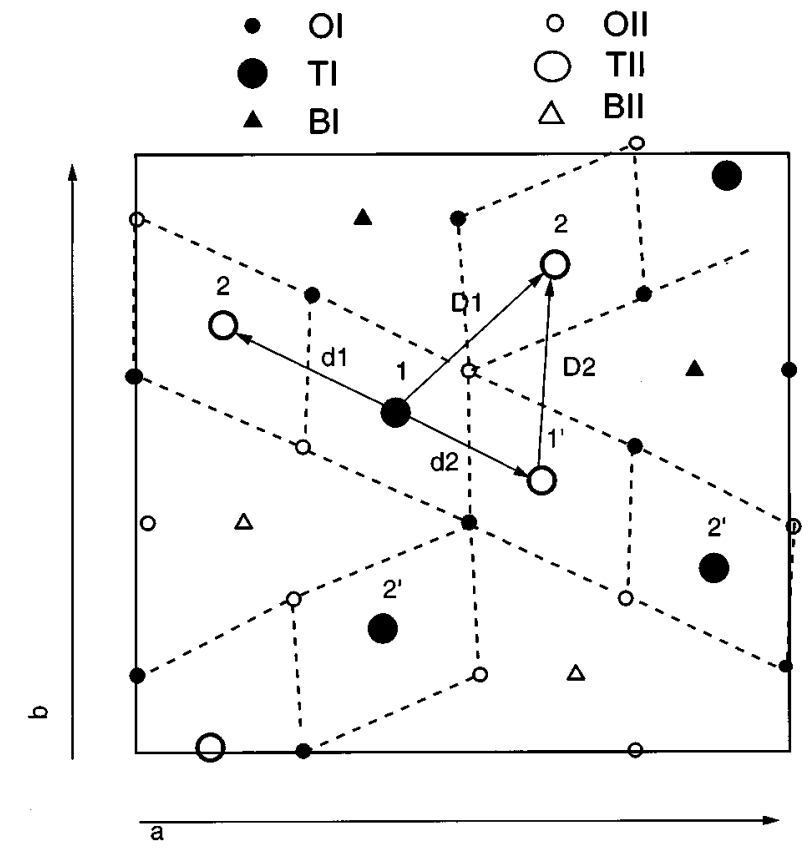

FIG. 1. A projection in the $a b$ plane of the structure of the warwickites. Sections of the ribbons are shown by dashed lines. The metals which lie in the center of octahedra are represented by big circles, the oxygens by smaller ones and the borons by triangles. The main interribbon coupling is due to superexchange coupling between metals in columns $1^{\prime}$ and 2 and 1 and $2^{\prime}$ in different ribbons mediated by the oxygen in the common corner of their different octahedra. Full and empty symbols refer to different $c$ axis coordinates.

Bragg-Brentano goniometer with monochromated $\mathrm{Cu} K \alpha$ radiation $(\lambda=1.5418 \AA)$. Measurements were performed with a scan step of $0.05^{\circ}$ in the $2 \theta$ range from 10 to $100^{\circ}$, with a fixed counting time of $4 \mathrm{~s}$. The program FULLPROF (Ref. 8) was used for the structure refinement. All the XRPD patterns were indexed to the space group Pnam (62), with crystallographic parameters similar to those previously reported, ${ }^{7}$ yielding the mean distances given in Table I. A typical result is displayed in Fig. 3, for the $\mathrm{ScMnOBO}_{3}$ sample. The good quality of the sample, with no sign of spurious phases, is suggested by the small intensities of the residual spectrum, also shown in Fig. 3.

The ac susceptibilities of these materials, measured in a commercial apparatus, together with those previously studied $^{1,2}$ are shown in Fig. 4. These data are plotted as $1 / \chi$ versus $T$. In every case we observe a Curie-Weiss law at high temperatures, $\chi \propto 1 /\left(T-\theta_{N}\right)$, with a negative intercept indicative of antiferromagnetic interactions. The values of $\theta_{N}$ are shown in Table II. As temperature is further de-

TABLE I. Structural parameters of the warwickite $\mathrm{ScMnOBO}_{3}$ (see Figs. 1 and 2 for the meaning of the distances). For the other warwickites studied in this work the distances above maintain the same relations. Furthermore the respective distances differ at most by $3 \%$ from those above.

\begin{tabular}{lccccc}
\hline \hline & $d_{1}(\AA)$ & $d_{2}(\AA)$ & $d_{3}(\AA)$ & $D_{1}(\AA)$ & $D_{2}(\AA)$ \\
\hline $\mathrm{ScMnOBO}_{3}$ & 3.304 & 2.892 & 3.298 & 3.657 & 3.359 \\
\hline \hline
\end{tabular}

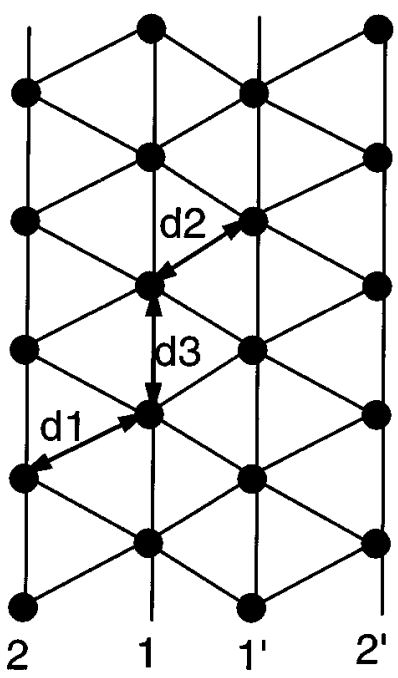

FIG. 2. Longitudinal section of a ribbon containing the metal sites. These sites form a triangular lattice where the cations are randomly located. There are three different distances between the metals which are given in Table I for $\mathrm{ScMnOBO}_{3}$.

creased there is a fluctuation regime starting at $T_{f}$ below which the susceptibilities are described by a power law $\chi \propto T^{-\alpha}$, characteristic of REHAC's. ${ }^{1,2}$ The exponents $\alpha$ $(\alpha<1)$ are also given in Table II as well as $T_{f}$. Excluding the system $\mathrm{MgTiOBO}_{3}$ we always observe a spin-glass transition at sufficiently low temperatures. The glassy nature of the transition at $T_{\mathrm{sg}}$ is indicated by the frequency dependence of the cusp in the susceptibility. Table II gives the transition temperatures $T_{\mathrm{sg}}$ for a frequency of $\nu=125 \mathrm{~Hz}$. Notice that for $\mathrm{MgCrOBO}_{3}$ and $\mathrm{MgFeOBO}_{3}$ the susceptibility continues to rise below $T_{\mathrm{sg}} \cdot{ }^{9}$

Mössbauer spectroscopy (MS) measurements were per-

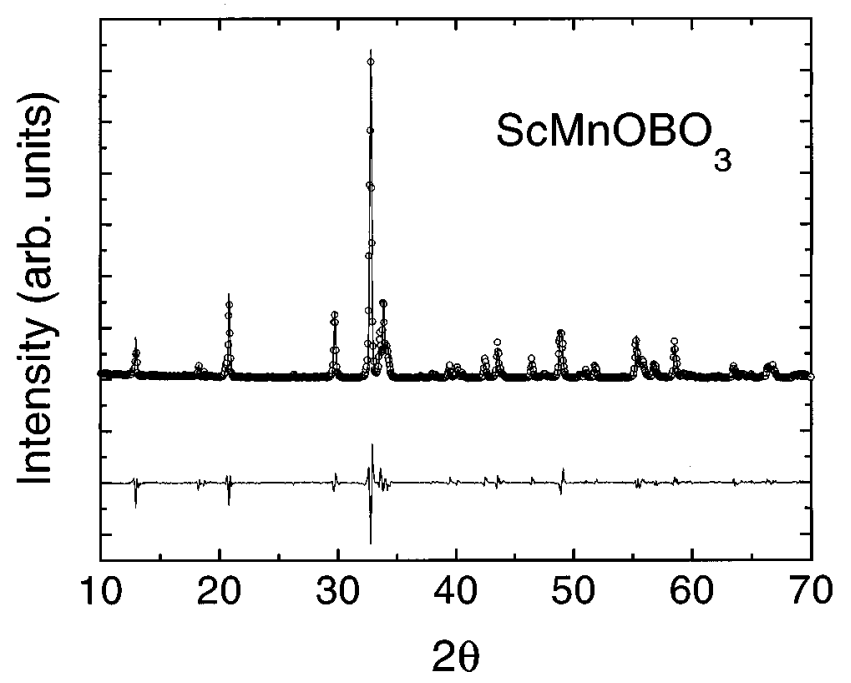

FIG. 3. Room-temperature x-ray powder-diffraction pattern for $\mathrm{ScMnOBO}_{3}$. The open circles represent the observed data. The solid line represents the calculated pattern obtained with the Rietveld refinement. The lower trace is a plot of the residual spectrum, observed minus calculated intensities. The resulting lattice parameters obtained from this data are $a=9.669(4) \AA$, $b=9.445(8) \AA$, and $c=3.297(9) \AA$. 

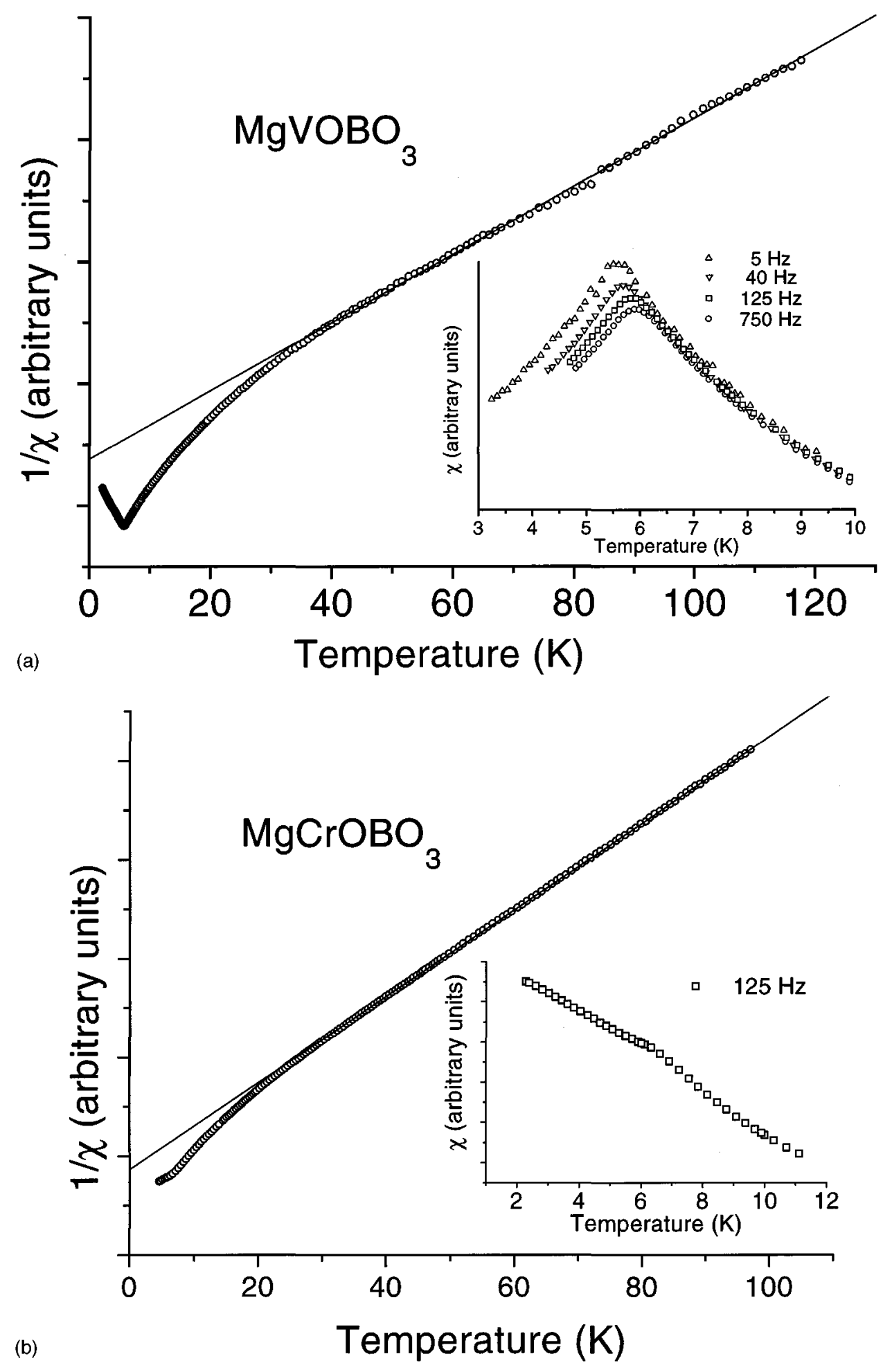

FIG. 4. The inverse of the ac susceptibilities of several warwickites as a function of temperature $\left(\nu=125 \mathrm{~Hz}, H_{0}=10 \mathrm{Oe}\right)$. The insets show the temperature dependence of the susceptibility in the neighborhood of the spin-glass transitions for different frequencies.

formed on $\mathrm{MgFeOBO}_{3}$ at temperatures between 300 and 4.2 $\mathrm{K}$. The spectra were obtained using a constant acceleration electromechanical drive system with a multichannel analyzer for collecting and storing the data. The temperature stability was better than $0.1 \mathrm{~K} .{ }^{57} \mathrm{Co}$ in rhodium was used at room temperature as a source, with nominal activity of $50 \mathrm{mCi}$. The hyperfine parameters for the paramagnetic spectra were obtained by a least-squares procedure assuming Lorentzian line shapes constrained to equal halfwidths. Following the Window model, the spectra obtained in the magnetically ordered regime were fitted to a hyperfine field distribution (HFD), $p(H)$, by superposing subspectra with Lorentzian line shapes, constrained to equal quadrupole splitting, isomer shift and halfwidths. ${ }^{10}$ Typical errors are $\pm 3 \%$ on hyperfine 

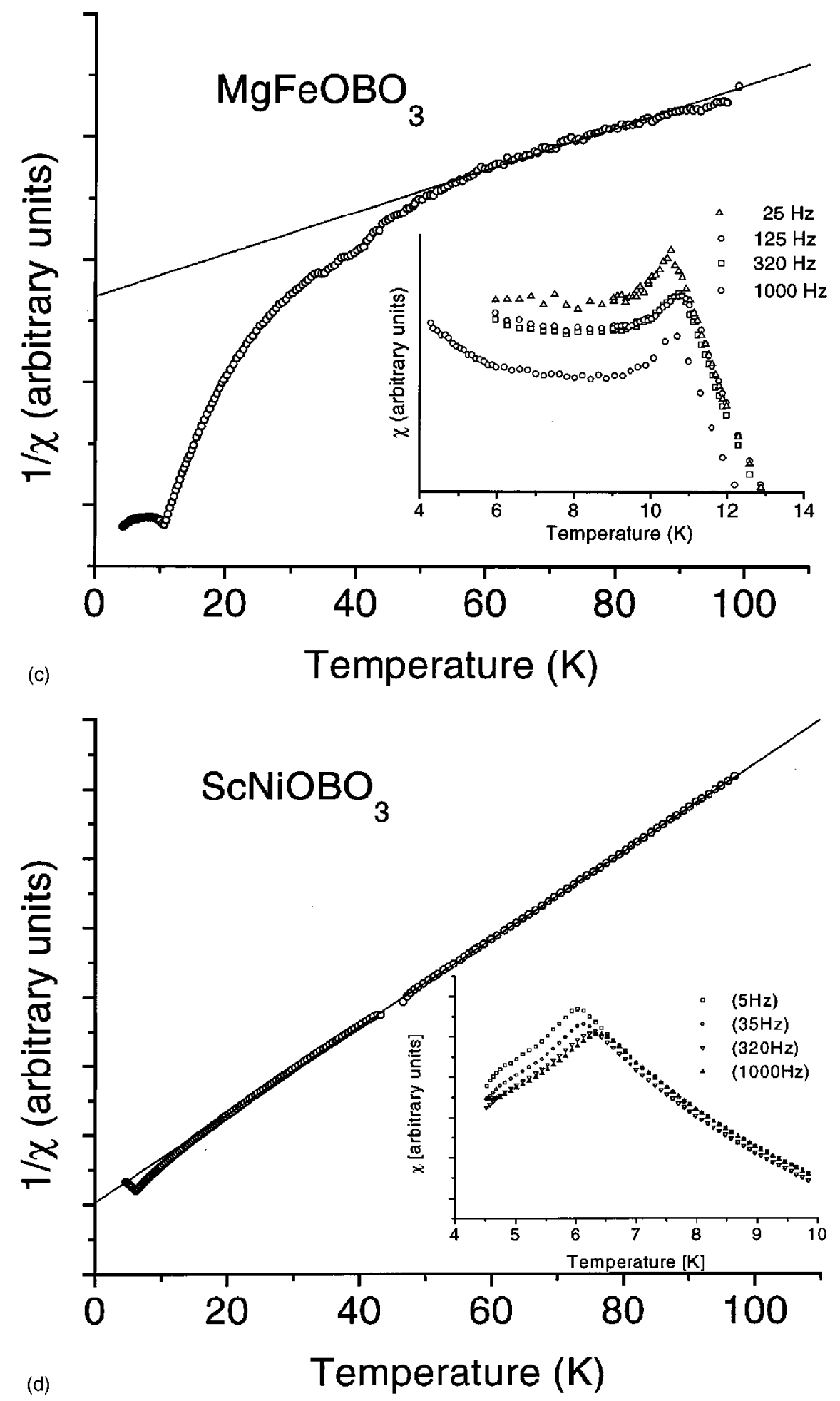

FIG. 4. (Continued).

parameters and $\pm 5 \%$ on site occupancies.

At room temperature the paramagnetic regime is represented by two symmetrical doublets (Fig. 5) with hyperfine parameters typical of high spin $\mathrm{Fe}^{3+}$ (Table III) and comparable to those previously published. ${ }^{13}$ The relative contributions, as measured from the spectral area, are quite similar to those reported: $:^{13} 78 \%$ from the largest doublet and $22 \%$ from the smallest. Following Wiedenmann et al. ${ }^{13}$ we attribute the largest doublet to $\mathrm{Fe}^{3+}$ in columns 1 and $1^{\prime}$ and the smallest to $\mathrm{Fe}^{3+}$ in columns 2 and $2^{\prime}$. This is very close to the occupancies found by Norrestam for $\mathrm{MgScOBO}_{3}$ in his crystallographic studies. ${ }^{6}$ As temperature decreases the paramagnetic pattern remains invariable up to temperatures about $15 \mathrm{~K}$, when a noticeable broadening of the spectrum is clearly observed, indicating relaxation effects.

The spectrum taken at $14 \mathrm{~K}$ shows clear evidence of HFD. The evolution for further decreasing temperature is characterized by an increase of the hyperfine magnetic fields 


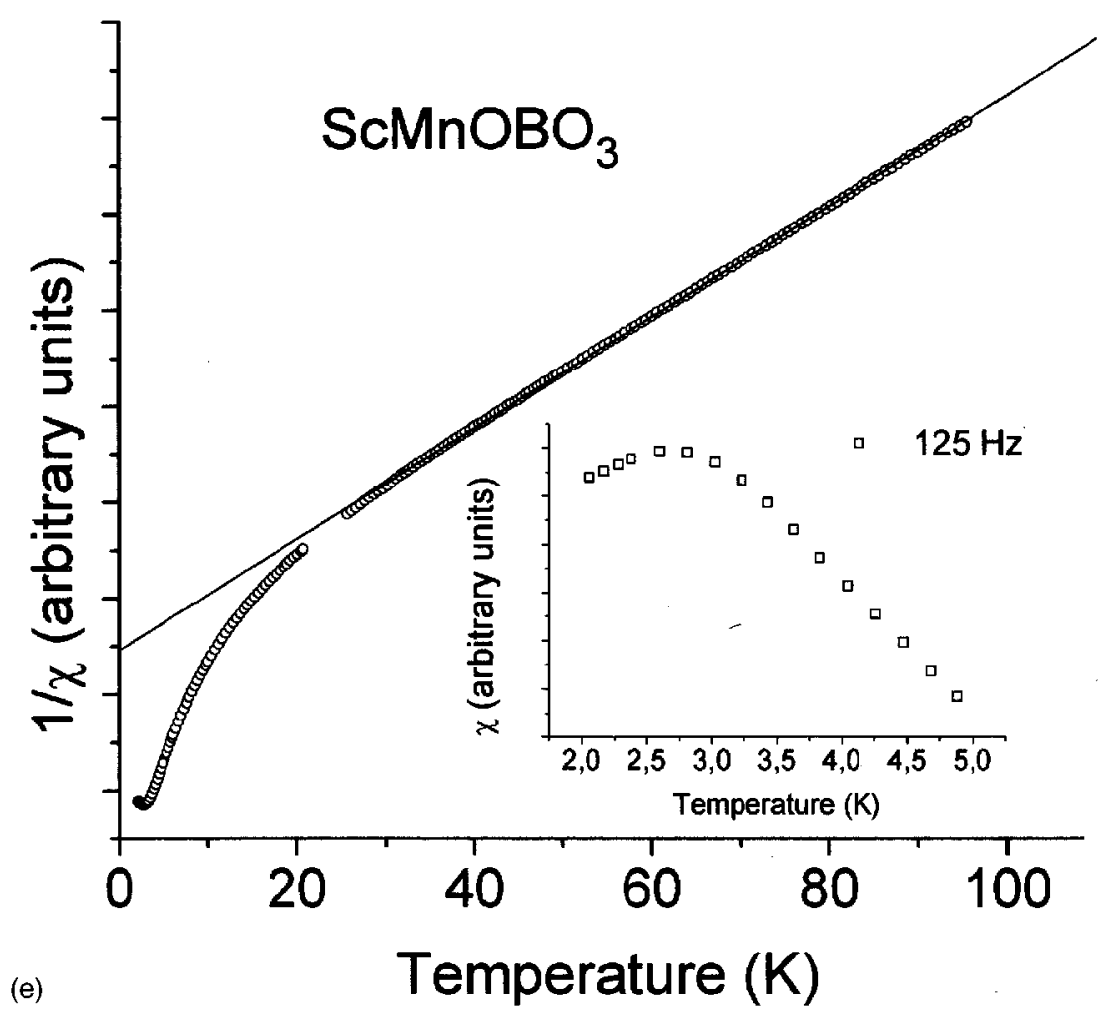

FIG. 4. (Continued).

and a decrease of the distribution halfwidths centered at the most probable hyperfine field. The spectrum taken at $4.2 \mathrm{~K}$ is quite similar to that reported by Wiedenmann et al., ${ }^{13}$ but we were not successful in appropriately fitting it to the parameters previously reported. A poor fitting was possible at the expense of using unreasonable linewidths. Therefore, it appears that a hyperfine field distribution is the best alternative to fit all the spectra taken below $15 \mathrm{~K}$. As illustrated in Fig. 6 , all the spectra taken below $15 \mathrm{~K}$ show $p(H)$ curves comprising four distributions, centered at fields displayed in Table IV. The $p(H)$ curve obtained at $14 \mathrm{~K}$ shows an almost resolved distribution centered at $71 \mathrm{kOe}$ and three unresolved ones centered at 177,271 , and $344 \mathrm{kOe}$. At $4.2 \mathrm{~K}$, the $p(H)$ with the most probable hyperfine field centered at 415 $\mathrm{kOe}$, and small contribution from the other distributions, is indicative that the system is very near the saturated state. About $97 \%$ of the iron atoms are magnetically coupled. The

TABLE II. Magnetic properties of some warwickites (see text). The effective moments obtained in the present work and in Ref. 13 are close to those obtained from the spin only values given above.

\begin{tabular}{lcccccc}
\hline \hline & $\theta_{N}(\mathrm{~K})$ & $J / k_{B}$ & $T_{f}(\mathrm{~K})$ & $\alpha$ & $T_{\text {sg }}(\mathrm{K})$ & Valence $(S)$ \\
\hline $\mathrm{MgTiOBO}_{3}$ & -73 & 73 & 20 & 0.83 & & $\mathrm{Ti}^{3+}(1 / 2)$ \\
\hline $\mathrm{MgVOBO}_{3}$ & -50 & 19 & 38 & 0.54 & 6 & $\mathrm{~V}^{3+}(1)$ \\
\hline $\mathrm{MgCrOBO}_{3}$ & -20 & 4 & 26 & 0.66 & 6.5 & $\mathrm{Cr}^{3+}(3 / 2)$ \\
\hline $\mathrm{MgFeOBO}_{3}$ & -278 & 23 & 100 & 0.54 & 11 & $\mathrm{Fe}^{3+}(5 / 2)$ \\
\hline $\mathrm{ScNiOBO}_{3}$ & -16 & 6 & 16 & 0.55 & 6 & $\mathrm{Ni}^{2+}(1)$ \\
\hline $\mathrm{ScMnOBO}_{3}$ & -60 & 5 & 26 & 0.50 & 2.7 & $\mathrm{Mn}^{2+}(5 / 2)$ \\
\hline \hline
\end{tabular}

spectrum taken at $10 \mathrm{~K}$ represents an intermediate regime between the onset of the magnetic ordering and the almost saturated state; the resulting $p(H)$, with four broad distributions, is consistent with such an intermediate regime.

These results resemble those obtained for other spin-glass systems. ${ }^{10-12}$ Following Window, ${ }^{10}$ we attributed the distribution centered at the lowest hyperfine field to iron atoms in columns 2 and $2^{\prime}$ and the distribution centered at higher fields to iron atoms in columns 1 and $1^{\prime}$ with different neighborhoods.

\section{DISCUSSION}

In Fig. 2 we show the longitudinal section of a ribbon containing the metal sites. The magnetic cations are randomly located on the sites of a triangular lattice. There are three different distances between the magnetic moments as shown in this figure and Table I. The distance $d_{2}$ among the magnetic ions in columns 1 and $1^{\prime}$ is such to make direct $d-d$ interaction important. ${ }^{4}$ On the other hand, for ions sepa-

TABLE III. Hyperfine parameters for $\mathrm{MgFeOBO}_{3}$ measured at $300 \mathrm{~K} . \Delta E_{Q}$ is the quadrupole splitting at the iron sites; $\delta_{\mathrm{Fe}}$ is the isomer shift relative to $\alpha$-Fe; $\Gamma$ is the linewidth at half-height; $A$ is the site occupancy, given by the relative spectral area. Typical errors are $\pm 3 \%$ on hyperfine parameters and $\pm 5 \%$ on site occupancies.

\begin{tabular}{ccccc}
\hline \hline$\Delta E_{Q}(\mathrm{~mm} / \mathrm{s})$ & $\delta_{\mathrm{Fe}}(\mathrm{mm} / \mathrm{s})$ & $\Gamma(\mathrm{mm} / \mathrm{s})$ & $A(\%)$ & Site assignment \\
\hline 0.84 & 0.34 & 0.35 & 78 & 1 and $1^{\prime}$ \\
\hline 0.78 & 0.50 & 0.29 & 22 & 2 and $2^{\prime}$ \\
\hline \hline
\end{tabular}


TABLE IV. Hyperfine parameters for $\mathrm{MgFeOBO}_{3}$ measured at low temperatures. $\Delta E_{Q}$ is the quadrupole splitting at the iron sites; $\delta_{\mathrm{Fe}}$ is the isomer shift relative to $\alpha$-Fe; $\Gamma$ is the linewidth at halfheight for all the Lorentzian used in the fitting; $H_{0}$ is the hyperfine field for isolated ${ }^{57} \mathrm{Fe} ; H_{1}, H_{2}$, and $H_{3}$ are attributed to iron atoms with different exchange interactions; $A_{i}$ is the relative area corresponding to the contribution of the hyperfine field $H_{i}$ to the total spectrum.

\begin{tabular}{lccc}
\hline \hline & $14 \mathrm{~K}$ & $10 \mathrm{~K}$ & $4.2 \mathrm{~K}$ \\
\hline$H_{0}(\mathrm{kOe})$ & 71 & 99 & 138 \\
\hline$A_{0}(\%)$ & 28 & 8 & 3 \\
\hline$H_{1}(\mathrm{kOe})$ & 177 & 226 & 224 \\
\hline$A_{1}(\%)$ & 46 & 27 & 3 \\
\hline$H_{2}(\mathrm{kOe})$ & 271 & 275 & 339 \\
\hline$A_{2}(\%)$ & 19 & 2 & 16 \\
\hline$H_{3}(\mathrm{kOe})$ & 344 & 350 & 415 \\
\hline$A_{3}(\%)$ & 7 & 63 & 78 \\
\hline$\Delta E_{Q}(\mathrm{~mm} / \mathrm{s})$ & -0.02 & -0.01 & -0.04 \\
\hline$\delta_{\mathrm{Fe}}(\mathrm{mm} / \mathrm{s})$ & 0.42 & 0.48 & 0.45 \\
\hline$\Gamma(\mathrm{mm} / \mathrm{s})$ & 0.48 & 0.44 & 0.45 \\
\hline \hline
\end{tabular}

rated by more than $3 \AA$, as in the case of $d_{2}$ and $d_{3}$, superexchange mediated by the oxygens in the shared edge of their different octahedra provides a competing mechanism for their interaction. ${ }^{4}$

From the values of $\theta_{N}$ in Table II we can derive the values of the antiferromagnetic exchange couplings $\left[\theta_{N}=2 z J S(S+1) / 3\right]$ shown also in this table. These values of $J$ are determined by the nearest-neighbor interaction among metals.

If the occupancies found by Norrestam in $\mathrm{MgScOBO}_{3}$ (Ref. 6) are generally valid for the warwickites, then for $\mathrm{ScNiOBO}_{3}$ and $\mathrm{ScMnOBO}_{3}$ most of the divalent magnetic cations are in the outer columns and at a distance $d_{3}$ apart.

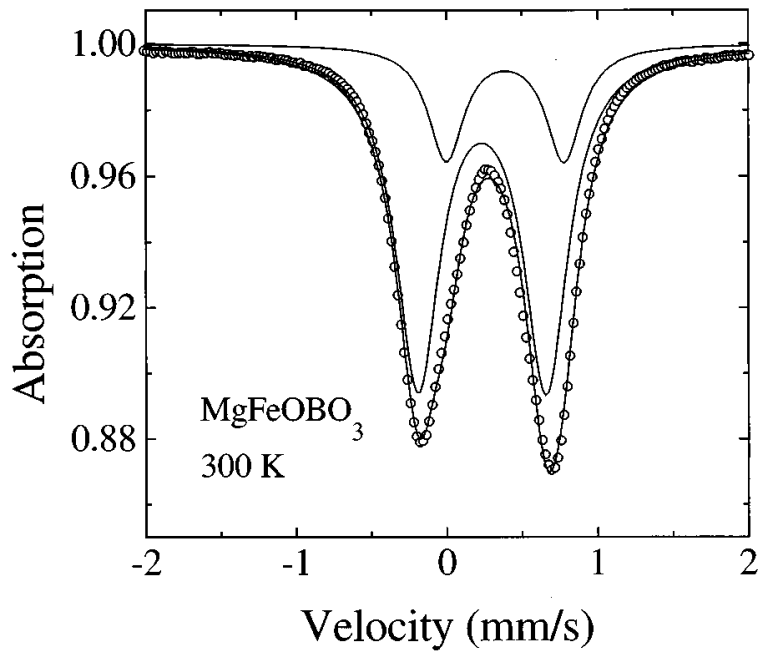

FIG. 5. Mössbauer spectrum for $\mathrm{MgFeOBO}_{3}$, taken at $300 \mathrm{~K}$.
These distances are larger than the corresponding nearestneighbor distance $d_{2}$ between trivalent magnetic ions located preferentially in the inner columns in the Mg systems. Consequently $J$ should be smaller for the Sc warwickites as is indeed the case (see Table II).

As temperature decreases the behavior of the susceptibility deviates, at $T_{f}$, from the simple Curie-Weiss behavior and the temperature dependence of the susceptibility for $T<T_{f}$ is described by the power law $\chi \propto T^{-\alpha}$. This behavior is characteristic of random exchange Heisenberg antiferromagnetic chains. ${ }^{14-16}$ In the present case the distribution of exchange interactions arises from the different distances and mechanisms of interaction between the magnetic ions randomly located in the ribbon. For sufficiently disordered antiferromagnetic Heisenberg chains we always expect such power-law behavior in the susceptibility to arise, independently of the value of the spin. ${ }^{16,17}$ Note that in the REHAC regime below $T_{f}$ the relevant energy scale is the cutoff of the exchange distribution which is determined by the distance $d_{2}$ for both $\mathrm{Mg}$ and $\mathrm{Sc}$ warwickites. In the present context the fluctuation temperature $T_{f}$ can be viewed as indicating the temperature at which the magnetic correlation length ${ }^{18}$ becomes of the order of the width of the ribbon and the confinement of the spins within this low dimensional structure starts to play a role. ${ }^{18}$

The main information about the interribbon interactions is contained in the spin-glass transition temperatures $T_{\text {sg }}$ given in Table II. These interactions are essentially due to superexchange among magnetic ions in columns 1 or $1^{\prime}$ and 2 in different ribbons. The distances $D_{1}$ and $D_{2}$ between cations
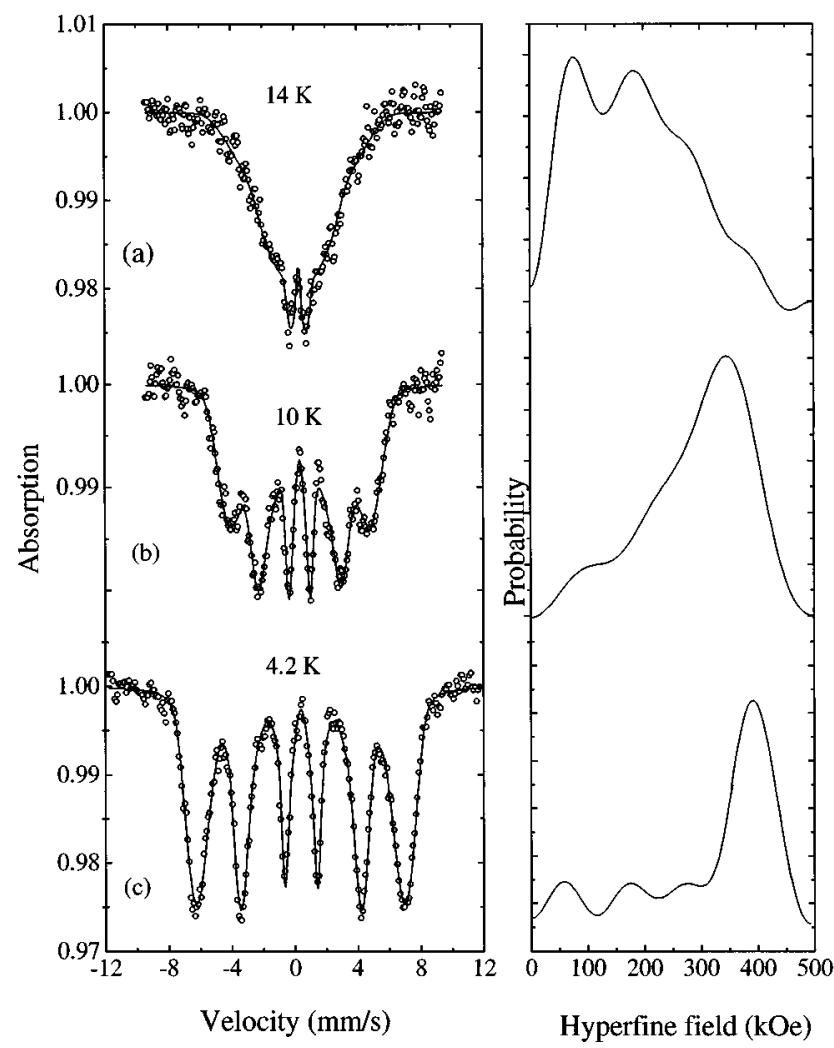

FIG. 6. Mössbauer spectra for $\mathrm{MgFeOBO}_{3}$, taken at (a) $14 \mathrm{~K}$, (b) $10 \mathrm{~K}$, and (c) $4.2 \mathrm{~K}$ as fitted by the hyperfine field distributions shown. 
in different ribbons are given in Table I. For $\mathrm{MgTiOBO}_{3}$ the corresponding angles cation-oxygen-cation are 112 and $117^{\circ}$. The superexchange in this case is mediated by the oxygens in the common corner of the octahedra containing the cations in the different ribbons (see Fig. 1). These interactions are smaller than the intraribbon direct $d$ - $d$ coupling as indicated by a direct comparison between the $\theta_{N}$ and $T_{\text {sg }}$. This hierarchy is a direct consequence of the structure of the warwickites and the reason for their one-dimensional magnetic behavior.

The results discussed above are consistent with those obtained from MS measurements on $\mathrm{MgFeOBO}_{3}$. Hyperfine magnetic splitting is observed for temperatures lower than 15 $\mathrm{K}$, i.e., $T_{\text {Möss }} \approx 14 \mathrm{~K}$. As for other spin-glass systems, ${ }^{12}$ $T_{\mathrm{sg}}<T_{\text {Mös }}$. Just above $T_{\text {Möss }}$ (e.g., $15 \mathrm{~K}$ ), relaxation effects are indicated by line broadening, and below this temperature the system relaxes within the MS time scale, yielding the hyperfine field splitting pattern. The $p(H)$ distribution obtained at $14 \mathrm{~K}$ indicates that about $28 \%$ of the iron atoms are decoupled at this temperature (Table IV). This proportion decreases to $8 \%$ at $10 \mathrm{~K}$, and to $3 \%$ at $4.2 \mathrm{~K}$. Since the contribution from the isolated iron atoms in columns 2 and $2^{\prime}$ is about $22 \%$ at $300 \mathrm{~K}$, the result obtained at $14 \mathrm{~K}$ means that some iron atoms in columns 1 and $1^{\prime}$ remain decoupled at the onset of the magnetic ordering. On the other hand, the $p(H)$ distribution obtained at $10 \mathrm{~K}$ with only $8 \%$ of the iron ions belonging to the lower field $p(H)$ is a clear evidence of the role played by the ions located in columns 2 and $2^{\prime}$ in the crossover to three-dimensional behavior.

The exponents $\alpha$ are similar and close to $\alpha \approx 1 / 2$ except for the $\mathrm{Ti}$ and $\mathrm{Cr}$ warwickites. For the former the more singular behavior of the susceptibility is probably associated with the spin $S=1 / 2$ of $\mathrm{Ti}$ for which we expect stronger quantum fluctuations. In fact it is only for $S=1 / 2$ that a random singlet phase is found independent of the amount of disorder in the exchange distributions. ${ }^{16}$ On the other hand, the absence of a spin glass transition for this compound is expected from the fact that the lower critical dimension $d_{c}$ for $S=1 / 2$ Heisenberg spin-glasses is larger than three. ${ }^{19}$ There are no studies available for quantum Heisenberg spin glasses with $S>1 / 2$ but it is natural to expect that fluctuations are less important as $S$ increases favoring the appearance of a transition.
An interesting feature in the data of Fig. 3 is that for $\mathrm{MgFeOBO}_{3}$ and $\mathrm{MgCrOBO}_{3}$ the susceptibility continues to rise below the transition temperature. This indicates that not all magnetic degrees of freedom are blocked at $T_{\mathrm{sg}}$. This is not surprising considering the high degree of frustration intrinsic to the triangular structure of the ribbons in the warwickite structure (see Fig. 2). The broad HFD obtained at 10 K (Fig. 6) together with the results of Table IV correlate quite well with this feature. Note that even at $4.2 \mathrm{~K}$ there is still a definite broadening of the distributions due to the random positions of the $\mathrm{Fe}$ ions in the ribbons and the different type of interactions between them. Such broadenings ruled out the possibility of fitting the $4.2 \mathrm{~K}$ spectrum with few magnetic sextets (for example, 4) and linewidth at halfheight lower than $0.50 \mathrm{~mm} / \mathrm{s}$.

\section{CONCLUSIONS}

We have presented results on the magnetic properties of warwickites. These materials have low-dimensional substructures in the form of ribbons which determine their magnetic properties. Since they can be formed with many transition metals a systematic investigation is possible. There are two types of magnetic couplings in the warwickites, namely intra- and interribbons. Antiferromagnetic couplings due to direct $d-d$ overlap and superexchange together with the random location of the cations in the ribbons give rise to the REHAC behavior observed for sufficiently high temperatures. The crossover to three-dimensional behavior which is associated with the spin-glass transitions is due to superexchange interactions between cations in different ribbons. The physics of the warwickites is dominated by low-dimensional effects, frustration, and disorder and has just begun to be understood.

\section{ACKNOWLEDGMENTS}

We would like to thank the Brazilian agencies CAPES, $\mathrm{CNPq}$, and FINEP for partial financial support. We also thank Professor E. Anda and Professor Maria Matos for useful discussions. We thank Professor Paulo H. Domingues for some preliminary results on $\mathrm{MgFeOBO}_{3}$ and Éder J. Kinast for help on the Rietveld refinements.
${ }^{1}$ J. C. Fernandes, R. B. Guimarães, M. A. Continentino, H. A. Borges, J. V. Valarelli, and A. Lacerda, Phys. Rev. B 50, 16754 (1994).

${ }^{2}$ M. A. Continentino, J. C. Fernandes, R. B. Guimarães, B. Boechat, H. A. Borges, J. V. Valarelli, E. Haanappell, A. Lacerda, and P. R. J. Silva, Philos. Mag. B 73, 601 (1996).

${ }^{3}$ M. Brunner, J-L. Tholence, L. Puech, J.J. Capponi, S. Haan, R. Calemczuk, J.C. Fernandes, and M.A. Continentino, Physica B 233, 37 (1997).

${ }^{4}$ M. Matos, R. Hoffman, A. Latge, and E. Anda, Chem. Mater. 8, 2324 (1996).

${ }^{5}$ D. C. Marcucci, A. Latge, E. Anda, M. Matos, and J. C. Fernandes (unpublished).

${ }^{6}$ R. Norrestam, Z. Kristallogr. 189, 1 (1989).
${ }^{7}$ J. J. Capponi, J. Chenavas, and J. C. Joubert, J. Solid State Chem. 7, 49 (1973).

${ }^{8}$ J. Rodriguez-Carvajal (1996): Short reference guide of the program FullProf. Version 3.1c, available in "pub/divers/fullp" of the anonymous ftp area of the LLB unix cluster.

${ }^{9}$ Similar features are seen in compounds with triangular structures; G. Aeppli, C. Broholm, A. Ramirez, G. P. Espinosa, and A. S. Cooper, J. Magn. Magn. Mater. 90\&91, 255 (1990).

${ }^{10}$ B. Window, J. Phys. C 3, S323 (1970); J. Phys. E 4, 401 (1971).

${ }^{11}$ M. S. Ridout, J. Phys. C 2, 1258 (1969).

${ }^{12}$ C. Meyer, F. Hartmann-Boutron, Y. Gros, and I. A. Campbell, J. Magn. Magn. Mater. 46, 254 (1985).

${ }^{13}$ A. Wiedenmann and P. Burlet, J. Phys. (Paris) Colloq. 8, C6-720 (1978); A. Wiedenmann, P. Burlet, and R. Chevalier, J. Magn. 
Magn. Mater. 15-18, 216 (1980).

${ }^{14}$ W. G. Clark, in Physics in One Dimension, edited by J. Bernasconi and T. Schneider, Springer Series in Solid State Science (Springer-Verlag, Berlin, 1981), p. 289.

${ }^{15}$ S. K. Ma and C. Dasgupta, Phys. Rev. B 22, 1305 (1980); J. E. Hirsch and J. V. Jose, ibid. 22, 5339 (1980).

${ }^{16}$ B. P. Boechat, A. Saguia, and M. A. Continentino, Solid State
Commun. 98, 411 (1996).

${ }^{17}$ R. A. Hyman and Kun Yang, Phys. Rev. Lett. 78, 1783 (1997).

${ }^{18}$ D. S. Fisher, Phys. Rev. B 50, 3799 (1994), and references therein.

${ }^{19}$ See, Heidelberg Colloquium in Glassy Dynamics, edited by J. L. van Hemmen and I. Morgenstern, Lecture Notes in Physics Vol. Vol. 23 (Springer-Verlag, Berlin, 1987). 